\title{
Looking at the dynamical heterogeneity in a supercooled polymer system through isoconfigurational ensemble
}

Cristian Balbuena, Melisa M. Gianetti, and Ezequiel R. Soulé

Citation: J. Chem. Phys. 149, 094506 (2018); doi: 10.1063/1.5039644

View online: https://doi.org/10.1063/1.5039644

View Table of Contents: http://aip.scitation.org/toc/jcp/149/9

Published by the American Institute of Physics

\section{Articles you may be interested in}

Glass forming phase diagram and local structure of Kob-Andersen binary Lennard-Jones nanoparticles

The Journal of Chemical Physics 149, 094502 (2018); 10.1063/1.5047465

Viscosity and self-diffusion of supercooled and stretched water from molecular dynamics simulations

The Journal of Chemical Physics 149, 094503 (2018); 10.1063/1.5042209

Possible universal relation between short time $\beta$-relaxation and long time $\alpha$-relaxation in glass-forming liquids The Journal of Chemical Physics 149, 024501 (2018); 10.1063/1.5033555

Comparison of single particle dynamics at the center and on the surface of equilibrium glassy films The Journal of Chemical Physics 149, 074501 (2018); 10.1063/1.5039505

Perspective: Highly stable vapor-deposited glasses

The Journal of Chemical Physics 147, 210901 (2017); 10.1063/1.5006265

Communication: Fast dynamics perspective on the breakdown of the Stokes-Einstein law in fragile glassformers

The Journal of Chemical Physics 148, 131102 (2018); 10.1063/1.5025614

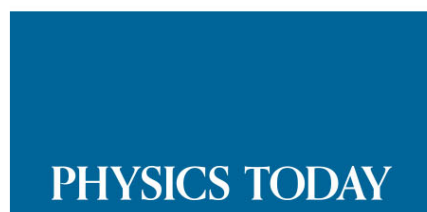

ADVANCED LIGHT CURE ADHESIVES

WHITEPAPERS
Take a closer look at what these environmentally friendly adhesive systems can do
READ NOW

PRESENTED BY

Q. MASTERBOND' 


\title{
Looking at the dynamical heterogeneity in a supercooled polymer system through isoconfigurational ensemble
}

\author{
Cristian Balbuena, ${ }^{\text {a) }}$ Melisa M. Gianetti, and Ezequiel R. Souléb) \\ Institute of Materials Science and Technology (INTEMA), University of Mar del Plata \\ and National Research Council (CONICET), J. B. Justo 4302, 7600 Mar del Plata, Argentina
}

(Received 9 May 2018; accepted 27 August 2018; published online 7 September 2018)

\begin{abstract}
The dynamic correlations that emerge in a polymer system in supercooling conditions have been studied using molecular dynamic simulations. It is known that when a glass former approaches the glass transition temperature, the dynamics of the system (in terms of the mobilities of the particles) not only significantly slows down but also becomes more heterogeneous. Several theories relate this slowing down to increasing spatial (structural) correlations, for example, through the onset of cooperative relaxation regions in the Adam-Gibbs theory. In this work, we employ Pearson's coefficient in the isoconfigurational ensemble (ICE) which allows us to study the dynamic correlations of the monomers in the ICE and establish the relation between the structure of the monomers and its dynamic behavior. Similar to what happens with mobility, monomers with highest correlation are clustered, and the clustering increases with decreasing temperature. An interesting result is that regions with high ICE dynamic correlation are not coincident with highly mobile or immobile regions. These results represent a new approach to the study of dynamic heterogeneity that emerges in glass forming liquids, complementing the more traditional characterization in terms of mobility. The methodology proposed in this work that characterize the connected dynamic regions to structural causes can represent an alternative way to observe the cooperative relaxation regions. Published by AIP Publishing. https://doi.org/10.1063/1.5039644
\end{abstract}

\section{INTRODUCTION}

After decades of research devoted to it, the glass transition is still not fully understood and remains a subject of great interest in the physics of condensed matter. ${ }^{1-3}$ One of the most striking features of the glass transition is that, as it is approached, there is a huge increase in viscosity (or equivalently $\alpha$-relaxation time) with relatively small decreases in temperature. Identifying the mechanisms that are responsible for the slowing down of the dynamics of supercooled liquids is still an open problem. ${ }^{4}$

There is general consensus that glass-forming liquids are dynamically heterogeneous, exhibiting a significant fraction of particles with extreme high or low mobility relative to the mean, whose positions are spatially correlated. Furthermore, experiments strongly suggest the occurrence of cooperative processes in glass-forming liquids, which, in turn, implies growing length scales that describe the spatial extent of correlated or cooperatively rearranging regions (CRRs), a term first used by Adam-Gibbs ${ }^{5}$ in their heuristic description of glassy dynamics. In particular, they proposed that reorganization in a liquid occurs via the CRRs, where the activation energy for relaxation is extensive in the number of atoms or molecules that make up the CRR. A qualitatively similar, but much more detailed, theoretical description is obtained within

a)Electronic mail: cbalbuena@ fi.mdp.edu.ar

b)Electronic mail: ersoule@ fi.mdp.edu.ar the framework of the random first order transition (RFOT) theory. ${ }^{6,7}$ At low temperatures, the glass-former is described as a mosaic of correlated domains that rearrange in a thermally activated, collective manner such that static and dynamic correlations coincide and grow with the viscosity. Derived from this mosaic scenario, the concept of amorphous order emerges in supercooled liquids. This structural order can be considered as the number of possible equilibrium configurations available to the liquid, which decreases when the temperature decreases.

One of the questions that still do not have a definitive answer is whether the glass transition is a purely dynamical phenomenon or if it is related to a thermodynamic transition (associated with a change in the structure). In large-scale computer simulations, it was observed that the most mobile clusters can be further divided into groups of atoms or molecules that move cooperatively in a roughly string-like fashion. ${ }^{8-11}$ The particles that present this dynamic behavior are identified as belonging to CRRs, and this describes the scenarios of the Adam-Gibbs and RFOT theory fairly well. ${ }^{11}$

Understanding the nature of the dynamical heterogeneities and how are they affected by the initial molecular configuration (structure) of the system might be the key to understanding the glass transition itself. The identification of a correlation between the structure and dynamic heterogeneity would allow us to discover the universal origin of slow dynamics in the glassy state. There have been many interesting studies in the last years trying to relate the heterogeneous dynamics with structural aspects, ${ }^{12-23}$ but there are also other 
approaches suggesting that structure is not dominant in the glass transition. ${ }^{24}$

In particular, the iso-configurational ensemble method (ICEM) ideated by Widmer-Cooper and Harrowel ${ }^{25}$ appears as a very useful tool for this objective. The method consists in running a large number of simulations, all of them with the same initial configuration but different initial velocities. In this way, any dynamic correlation existing in the different runs of the ICE must have a structural origin, as the initial configuration is the only thing in common between the trajectories. This method was applied to systems of attractive spheres and allowed to identify regions of high mobility and low mobility that are defined by the initial configuration, as well as the presence of correlations (between different trajectories of the ICE, measured through Pearson's correlation coefficient) in the displacement of neighbouring particles. ${ }^{26-28}$ Near the $T_{g}$, the spatial heterogeneities are immediately evident in the isoconfigurational displacement (or propensity) field. Surprisingly, however, simple structural quantities, such as free volume and local potential energy, show little correlation with the heterogeneity in the propensity. ${ }^{29}$ In some models, localized soft modes appear to correlate strongly with propensity at short time scales. ${ }^{30}$

Other approach, more specific in terms of structure, suggests that particles with low mobility or propensity tend to be found in certain locally preferred structures (LPS) with an increase in supercooling. ${ }^{31-33}$ A striking result is that the relation between LPS and dynamical slowdown is highly system dependent. ${ }^{34}$ Consequently, this approach seems to offer little hope of attaining a universal theory of glass transition.

An alternative approach to those cited is the point-to-set method. ${ }^{35}$ Among the interesting results obtained with this technique is the finding that the order-agnostic $\mathrm{PtS}$ correlation length grows faster than simpler static lengths in various glassforming liquids as $\mathrm{Tg}$ is approached, supporting the RFOT scenario. ${ }^{35-39}$ Nevertheless, while both lengths, the dynamic as determined by the four-point correlation length $\xi_{4}$ and the static $\xi_{P t S}$, seem to universally increase upon cooling, the relation between the two remains unclear, and in the numerically accessible regime over which they have been determined, they grow at different rates, the former more rapidly than the latter. Kob et al. ${ }^{40}$ found a non-monotonic behavior of a dynamic correlation length around $T_{M C T}$. Recently a nonmonotonic evolution of dynamical correlations in colloidal liquids ${ }^{41}$ was unveiled. If, as these results show, $\xi_{4}$ exhibits a different scaling with temperature upon approaching the glass transition, a coincidence of structural and dynamic lengths at low temperature is possible, although this approach is also controversial. $^{42,43}$

Another possible solution to the discrepancy observed between static and dynamic lengths could be that $\xi_{4}$ is not representative of dynamic lengthscales. Recently Dunleavy et al., ${ }^{44}$ through the mutual information in the ICE displacement probability distributions of pairs of particles, found two modes of correlated motion associated with distinct geometric motifs and local density. The dynamic correlation lengths that they computed by an information theoretic approach are similar to those found directly from structural quantities.
The aim of this work is to study dynamical heterogeneities and correlations between particles for a coarse-grained polymer model. We study the dynamic correlations that emerge in an isoconfigurational assembly but considering relative movements of each particle with respect to its propensity. This dynamic correlation was calculated by the Pearson correlation coefficient, which reflects the degree of displacement correlation between specific pairs of particles through all the runs of the ICE and like the propensity for motion has a structural correlation. Moreover, the chain connectivity in polymeric materials is an extra factor that strongly affects the dynamical behavior, so understanding the glass transition in these systems poses an extra challenge. ${ }^{45}$ The results of this work show that this magnitude presents a behavior very similar to the non-Gaussian parameter. It was found that particles with highest correlation are clustered like the mobile and immobile particles but do not integrate the same clusters. This approach promotes an alternative way of observing dynamic heterogeneities.

\section{METHODOLOGY}

In this section, the details of the simulation model and computational methods for the dense bead-spring polymer melt are described. The polymer molecules are modeled by soft-core spheres and stretching/bending springs with freely rotating chains (FRC). ${ }^{46,47}$ All monomers interact through the Lennard-Jones potential,

$$
U\left(r_{i j}\right)=4 \epsilon\left[\left(\frac{\sigma}{r_{i j}}\right)^{12}-\left(\frac{\sigma}{r_{i j}}\right)^{6}\right],
$$

where $r_{i j}$ is the distance between the beads $i$ and $j, \sigma$ is the distance at which the interparticle potential is zero, and $\epsilon$ is the depth of the potential at the minimum. Both parameters take the value of $1 . U\left(r_{i j}\right)$ is truncated for $r_{i j}$ greater than $2.0 \sigma$ with the long-tail correction applied. In addition, the bonded neighbors in a chain interact through the finitely extensible nonlinear elastic (FENE) bond potential $U_{\text {bond }}$,

$$
U_{\text {bond }}\left(r_{i j}\right)=-15 R_{0}^{2} \ln \left[1-\left(r_{i j} / R_{0}\right)^{2}\right],
$$

where $R_{0}=1.5 \sigma$ is the maximum length of the bond.

Simulations were implemented using the open-source software LAMMPS. ${ }^{48}$ The time step for integration was $\mathrm{dt}=0.01 \tau$, where $\tau$ is the unit of time $\tau=\left(m \sigma^{2} / \epsilon\right)^{0.5}$ and $m$ is the mass of the bead $(m=1)$. The Nosé-Hoover thermostat and Andersen barostat were used to control the temperature $\mathrm{T}$ and the pressure $\mathrm{P}$ of the system, respectively. For all the simulations in this study, the pressure of the system was set to zero $(P=0)$. Our results are primarily based on molecular dynamics (MD) simulations of a melt containing 133 chains of bead-spring polymers, each chain containing 30 monomers. This chain length represents a non-entangled polymer. In some cases, systems with 2000 chains were analyzed in order to improve the statistics.

The system was equilibrated by generating runs of 100 $\tau_{\alpha}(\mathrm{T})$ in the NPT ensemble and then $100 \tau_{\alpha}(\mathrm{T})$ in the NVT ensemble. Finally, NVT and NVE were alternated for a period of $20 \tau_{\alpha}(\mathrm{T})$. This was done at each temperature studied. Full 
equilibration at each temperature was verified by the absence of drift in thermodynamic magnitudes and by the absence of aging. The NVE ensemble was used for production runs and for latter production of the ICEM. For $T>0.47$, every system under study can be equilibrated within a reasonable computation time, but the lowest temperature studied in this work was 0.50 , limited by the computation time required by the ICEM. At each studied temperature, 15 ICEs were generated, each one starting from equilibrated configurations obtained from independent trajectories. The ICE consisted of 500 trajectories.

\section{RESULTS AND DISCUSSION}

\section{A. Characteristic times}

First, two dynamic characteristic times of a glass former, the structural relaxation time, $\tau_{\alpha}$, and the time of maximum dynamical heterogeneity, $t^{*}$, are calculated.

In order to determine $\tau_{\alpha}$, the incoherent intermediate scattering function is calculated, which is defined as

$$
F_{s}\left(q_{0}, t\right)=\frac{1}{N} \sum_{i=1}^{N}\left\langle\exp \left[i q_{0}\left(\left|\mathbf{r}_{i}(t)-\mathbf{r}_{i}(0)\right|\right)\right]\right\rangle,
$$

where $\mathrm{N}$ is the number of monomers and $q_{0}$ is the wavenumber corresponding to the first peak of the $g(r)$ function. Figure 1 shows this function at different temperatures. At high temperature, it behaves as a single decaying exponential (single relaxation mechanism), but at lower temperatures (close to $\mathrm{Tg}$ ), there is a two-step decay: a short time relaxation ( $\beta$ relaxation) and a long time relaxation (structural or $\alpha$ relaxation). This is a characteristic behavior of glass-formers in the supercooled regime. ${ }^{49}$ One of the main achievements of MCT was the ability to predict this behavior. ${ }^{50}$ Relaxation times are defined as $F\left(q_{0}, \tau_{\alpha}\right)=e^{-1}$.

Dynamic heterogeneity can be related to a non-Gaussian distribution of particle mobility ${ }^{51}$ high-mobility particles (relaxing in a time scale of $t^{*}$ ) are associated with diffusion, while low mobility particles (time scale of $\tau_{\alpha}$ ) are related to

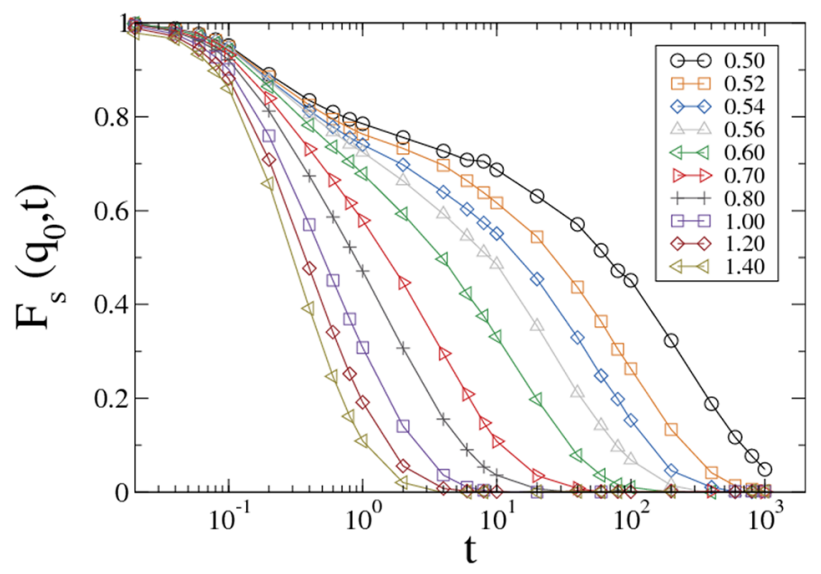

FIG. 1. Incoherent intermediate scattering function at different temperatures. At high temperatures, a single relaxation mechanism is observed, while at lower temperatures, two different relaxation processes exist ( $\alpha$ and $\beta$ ). Structural relaxation time is defined as the time that $F\left(q_{0}, \tau_{\alpha}\right)=e^{-1}$, where vector $q_{0}$ corresponds to the first peak of the structure factor. viscosity. ${ }^{11}$ Time $t^{*}$, which is smaller than $\tau_{\alpha}$, represents the time at which heterogeneity in particle mobility is the largest. Several ways to define $\mathrm{t}^{*}$, based in different parameters, have been used in the literature, but they all give a similar result. In this work, we adopt the definition that $t^{*}$ corresponds to the time when the non-Gaussian parameter, defined as

$$
\alpha_{2}=\frac{3\left\langle r^{4}(t)\right\rangle}{5\left\langle r^{2}(t)\right\rangle^{2}}-1
$$

is maximum. For a Gaussian process, $\alpha_{2}$ is strictly zero and a non-zero value implies a non-trivial (i.e., heterogeneous) dynamics. Figure 2 shows $\alpha_{2}$ as a function of time for the average of all monomers, at different temperatures. The same behavior is observed in every case: at small times, ballistic movement, which has a Gaussian distribution, is observed and $\alpha_{2}(t)$ is zero. For intermediate times, $\alpha_{2}(t)$ rises, reaching a maximum (this time is defined as $t^{*}$ ). Eventually, at long enough times, monomer movement reaches a diffusive regime and $\alpha_{2}(t)$ decreases approaching zero. It can be seen in Fig. 2 that when temperature decreases, $\alpha_{2}$ is larger (heterogeneity increases), and the maximum is observed at larger time ( $t^{*}$ increases). This behavior is observed in many glassformers. It was recently suggested that the breakdown of the time-temperature superposition (TTS) near the glass transition temperature is related to the dynamic heterogeneity. ${ }^{47}$ Figure 3 shows both characteristic times, $\tau_{\alpha}$ and $t^{*}$, as a function of temperature. The typical behavior of a glass former is observed: at high temperature, both times converge (homogeneous dynamics), and when temperature decreases, $\tau_{\alpha}$ becomes larger than $t^{*}$. The increase in relaxation times is more significant at lower temperatures when the glass transition is approached.

\section{B. Monomer propensity for motion}

By using ICEM, the effect of the initial structure on the dynamics of individual particles can be assessed. ${ }^{25}$ To this end, the dynamic propensity for motion is defined as the ICEaverage displacement of monomer $i$ at time $t$ in the total number of trajectories in the ICE $\left(N_{I C}\right)$,

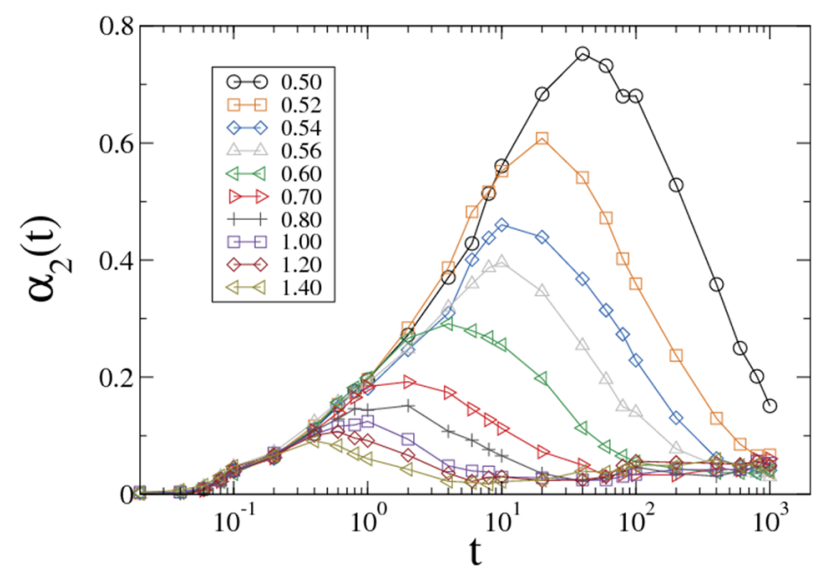

FIG. 2. Non-Gaussian parameter $\alpha_{2}(t)$ at different temperatures. The time of maximum dynamic heterogeneity $t^{*}$ is defined as the time when a $\alpha_{2}(t)$ is maximum. 


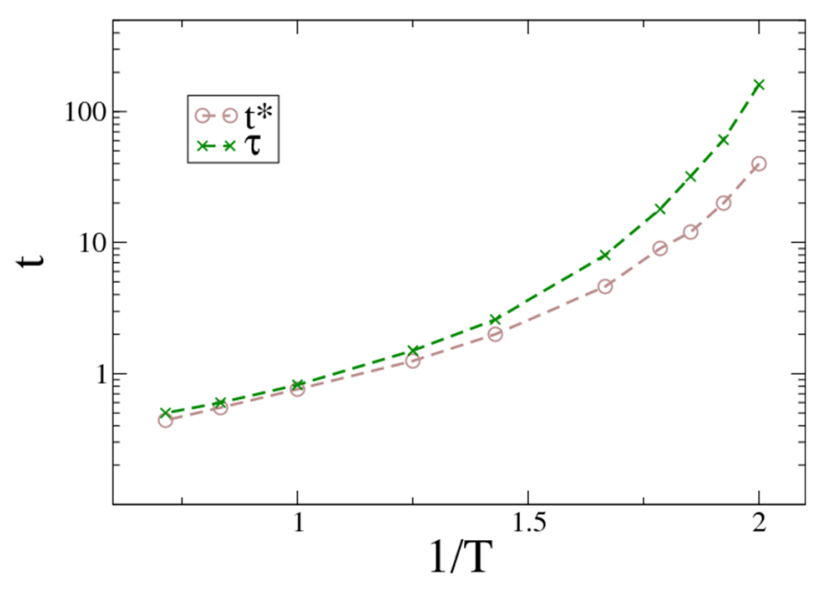

FIG. 3. Characteristic times, $\tau_{\alpha}$ and $t^{*}$, as a function of temperature.

$$
\left\langle\Delta r_{i}^{2}(t)\right\rangle_{I C}=\frac{1}{N_{I C}} \sum_{w=1}^{N_{I C}}\left|\mathbf{r}_{i}(w, t)-\mathbf{r}_{i}(0)\right|^{2},
$$

where $w$ corresponds to a certain trajectory of the ICE and $\mathbf{r}_{i}(0)$ is the position of particle $i$ in the initial configuration of the ICE. Figure 4 shows the average monomer propensity as a function of time at different temperatures. Propensity behaves very similarly to mean square displacement (MSD), as the former is the squared-displacement of a particle averaged over the ICE and the latter is the squared-displacement over all particles in a single run. It is observed that the displacement of a single particle through the different runs of the ICE shows an important dispersion. Figure 5 shows the frequency distribution of the displacement of each monomer through the different runs of the ICE at $t^{*}$. As the temperature decreases, the distribution becomes more asymmetric. This means that the heterogeneous dynamics manifests not only as a spatial heterogeneity, but also the dynamics of a given monomer in the different runs is heterogeneous. ${ }^{28}$

\section{Monomer dynamic correlation}

Dynamic correlations between particles in the isoconfigurational ensemble must have a structural origin and must be related to the initial particle configuration as this is the only

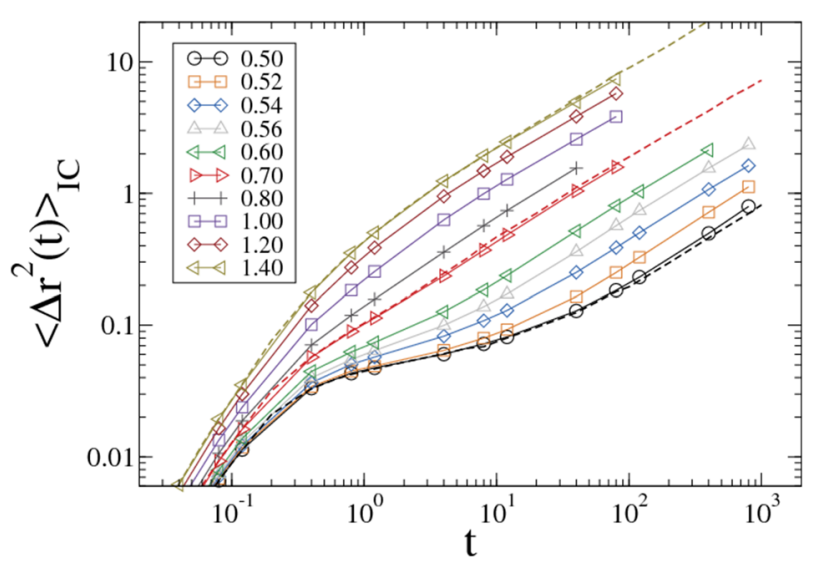

FIG. 4. ICE Average propensity at different temperatures, as a function of time. MSD at $0.50,0.70$, and 1.4 are also shown (dashed line).

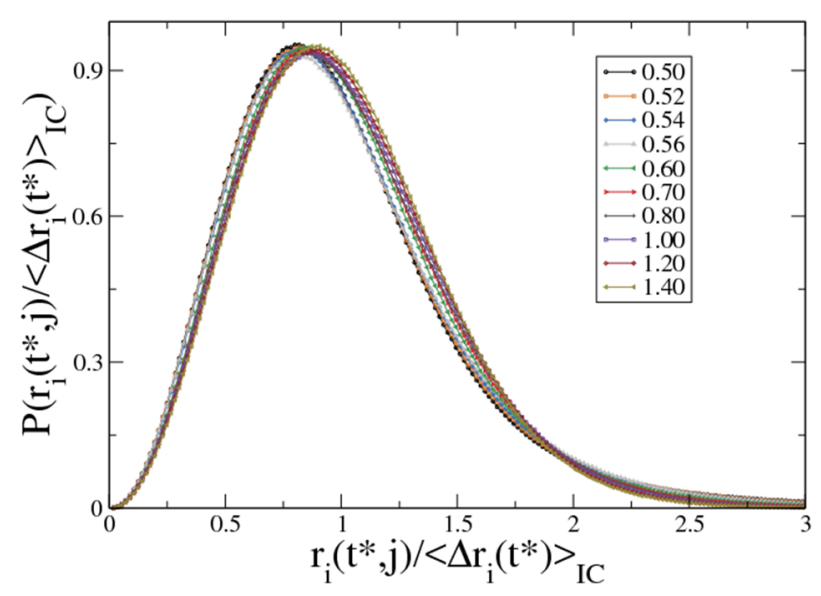

FIG. 5. Frequency distribution of the displacement of each particle, normalized with respect to its propensity, at $\mathrm{T}=0.54$ and $\mathrm{t}=t^{*}$.

thing in common between the different trajectories of the ICE. In order to analyze dynamic correlations over the whole set of ICE trajectories, the Pearson correlation coefficient between monomer pairs $\left(k_{i j}\right)$ was used. The value of $\left(k_{i j}\right)$ for two particles that are separated by a distance $r_{i j}=\left|\mathbf{r}_{i}(0)-\mathbf{r}_{j}(0)\right|$ in the initial configuration is defined as follows:

$$
k_{i j}\left(r_{i j}, t\right)=\frac{1}{S_{i}(t) S_{j}(t)} \sum_{w=1}^{N_{I C}} \psi_{i}(w, t) \psi_{j}(w, t),
$$

where $\psi_{i}(w, t)=\mid\left(\mathbf{r}_{i}(w, t)-\mathbf{r}_{i}(0) \mid-\left\langle\Delta r_{i}(t)\right\rangle_{I C}\right.$, and $S_{i}(t)$ is the standard deviation in the displacement of the monomer $i$ at time $t$ in the ICE.

Pearson's coefficients can take values between -1 and +1 , and it is a measure of the correlation between displacement of two monomers, relative to the ICE average displacement (propensity) of each monomer. ${ }^{28}$ A negative correlation coefficient between two monomers implies that a large relative movement of one monomer is conditioned to a small relative movement of the other. A positive correlation implies that both monomers tend to present simultaneously large or small relative movement. A small value of the correlation coefficient implies that the relative movement of the monomers is independent of each other. In a polymer, not every pair of monomers is the same, as both monomers can belong to the same polymeric chain or to different chains. Moreover, if two monomers belong to same chain, they can be bonded, or they can be separated by a number of bonded monomers. Two types of correlations were considered: inter-chain correlations between monomers that belong to different chains, identified as $A B$, and correlations between monomers, including intra-chain, identified as $A A$. The analysis of $A B$ correlations eliminates the trivial higher correlation between monomers in the same chain, and the comparison of both allows us to identify the effects of chain connectivity.

Figure 6 shows the behavior of $k_{i j}(r, t)$ at the different characteristic times, $t^{*}$ and $\tau_{\alpha}$, as a function of distance, at a temperature of 0.54 , for the $A B$ case. For every distance, the correlation at $t^{*}$ is higher than at $\tau_{\alpha}$. This difference is higher at the nearest- and second-nearest neighbour 


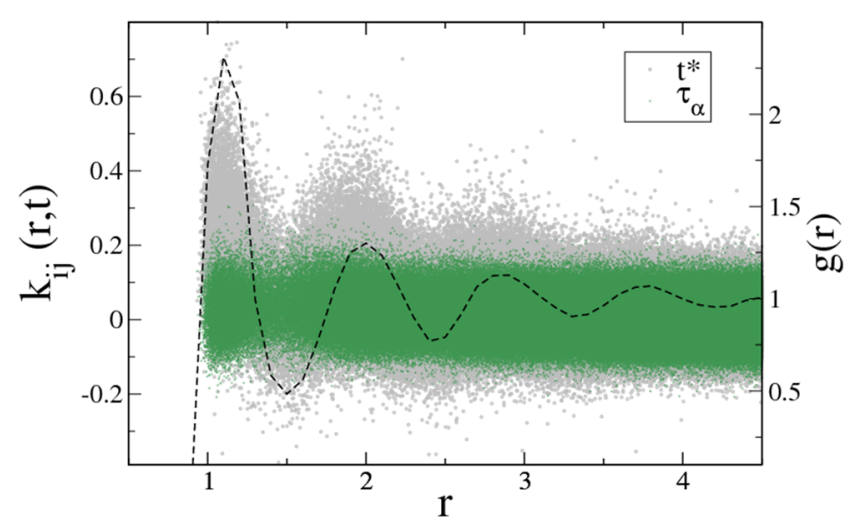

(a)

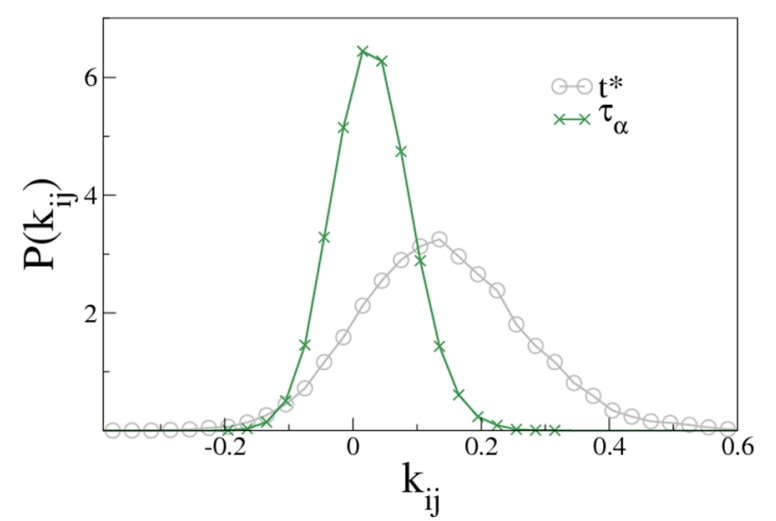

(b)

FIG. 6. (a) $k_{i j}$ at $\mathrm{T}=0.54$ as a function of distance, calculated at the two characteristic times, $t^{*}$ and $\tau_{\alpha}$. Only monomers belonging to different polymer chains $(\mathrm{AB})$ are considered. The Radial distribution function, $\mathrm{g}(\mathrm{r})$, is included in the graph (dashed line). (b) Distribution of $k_{i j}$ at the nearest-neighbour distance for both characteristic times.

distance. This analysis shows that the effect of the structure (initial configuration) on dynamic correlations is observed at a time scale of $t^{*}$, while at $\tau_{\alpha}$, there is no significant structureconditioned correlation. This can be expected, considering that the time scale $\tau_{\alpha}$ is related to structural relaxation, such that the system forgets the initial configuration. In addition, it can be observed that the correlation at $t^{*}$ is larger for nearestneighbours, which is reasonable considering that at this time the prevailing dynamic is the cage regime, where each particle interacts with its nearest neighbours. By looking at the distribution of $k_{i j}$ for $r_{i j}<1.5$, shown in Fig. 6(b), it can be observed that the distribution curve at $t^{*}$ is placed at larger values of $k_{i j}$ compared to $\tau_{\alpha}$, and the mean value is not zero. The curve for $\tau_{\alpha}$ is zero for $k_{i j}>0.25$, while for $t^{*}$, it is zero for $k_{i j}>0.58$.

Having the values of $k_{i j}$ for every pair of monomers, an average global Pearson's correlation is calculated as

$$
K(r, t)=\frac{1}{\rho(r)} \sum_{i \neq j}^{N} k_{i j}\left(r_{i j}, t\right) \delta\left(r-\left|r_{i j}\right|\right),
$$

where $\rho(r)=\sum_{i \neq j}^{N} \delta\left(r-r_{i j}\right)$. The magnitude $K(r, t)$ reflects the global dynamic correlation between one monomer and all the monomers placed at a distance $r$. Figure 7 shows the behavior of $K\left(r, t^{*}\right)$ at different temperatures for $A A$ and $A B$ cases. In both of them and at every temperature, $K\left(r, t^{*}\right)$ oscillates as
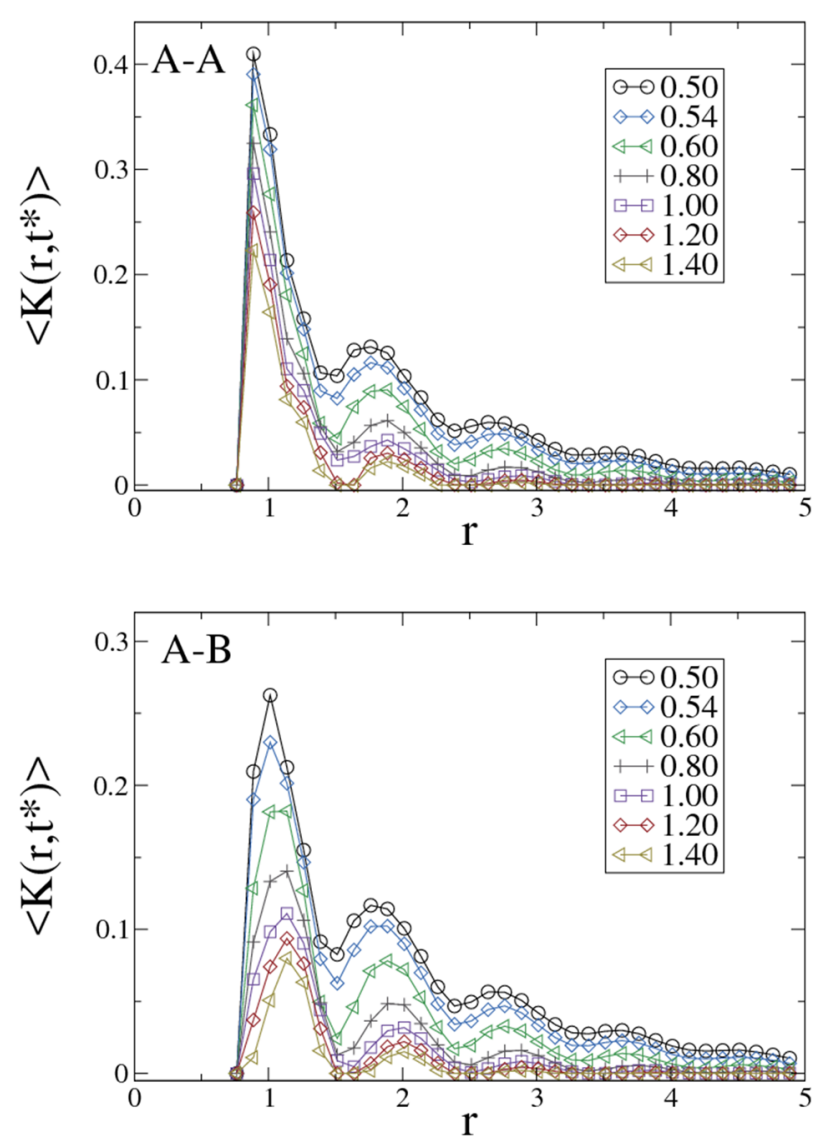

FIG. 7. Global correlation as a function of distance for cases $A A$ and $A B$ at $t^{*}$ and different temperatures.

a function of distance, following the oscillations of the radial distribution function (RDF), shown in Fig. 8. It can be observed that correlation increases when temperature decreases. At high temperatures, $(T>1.0), K\left(r, t^{*}\right)$ approaches zero for $r_{i j}>2.5$. Instead, at lower temperatures and as $\mathrm{Tg}$ is approached, the correlation is significant at larger distances. The difference between the $\mathrm{AB}$ and $\mathrm{AA}$ case is that there is a higher correlation at the nearest-neighbour distance for AA, as expected due to connectivity, but for larger distances, $K\left(r, t^{*}\right)$ for both cases is very similar. This reflects that the correlation at $t^{*}$ is essentially

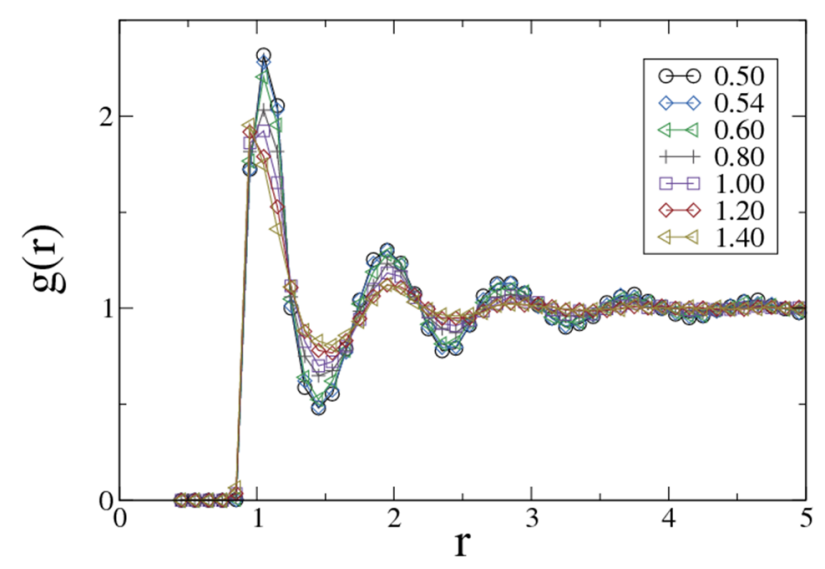

FIG. 8. Radial distribution function, considering all the monomers, at different temperatures. 
intermolecular. Analyzing how the radial distribution function $\mathrm{g}(\mathrm{r})$, Fig. 8 , and $K\left(r, t^{*}\right)$ depend on the distance, it can be noted that when oscillations in $\mathrm{g}(\mathrm{r})$ vanish, $K\left(r, t^{*}\right)$ decays to zero. This establishes the close relationship between the structuring of the system and the dynamic correlation of the particles.

\section{Nearest-neighbour dynamic correlation as a function of time}

The time evolution of the global dynamic correlation at the nearest-neighbour distance, $K(t)$, is shown in Fig. 9. The behavior of the $A B$ case is similar to that of the non-Gaussian parameter, in that it approaches zero both for short (approaching zero) and long times $\left(t>\tau_{\alpha}\right)$. In the $A A$ case, the correlation for large times does not go to zero, instead it increases; this is due to the movement of the whole polymer chain. The time at which $K(t)$ is maximum is very close to $t^{*}$. These results indicate that correlative movement and dynamical heterogeneity seem to be related. This correlative movement, as it is observed in ICEM, indicates a dynamic correlation dictated by the structure.

Having defined a measurement of monomer mobility, which is the propensity for movement, and a movement correlation between nearest neighbours through the Pearson coefficient, the question arises whether both magnitudes are related
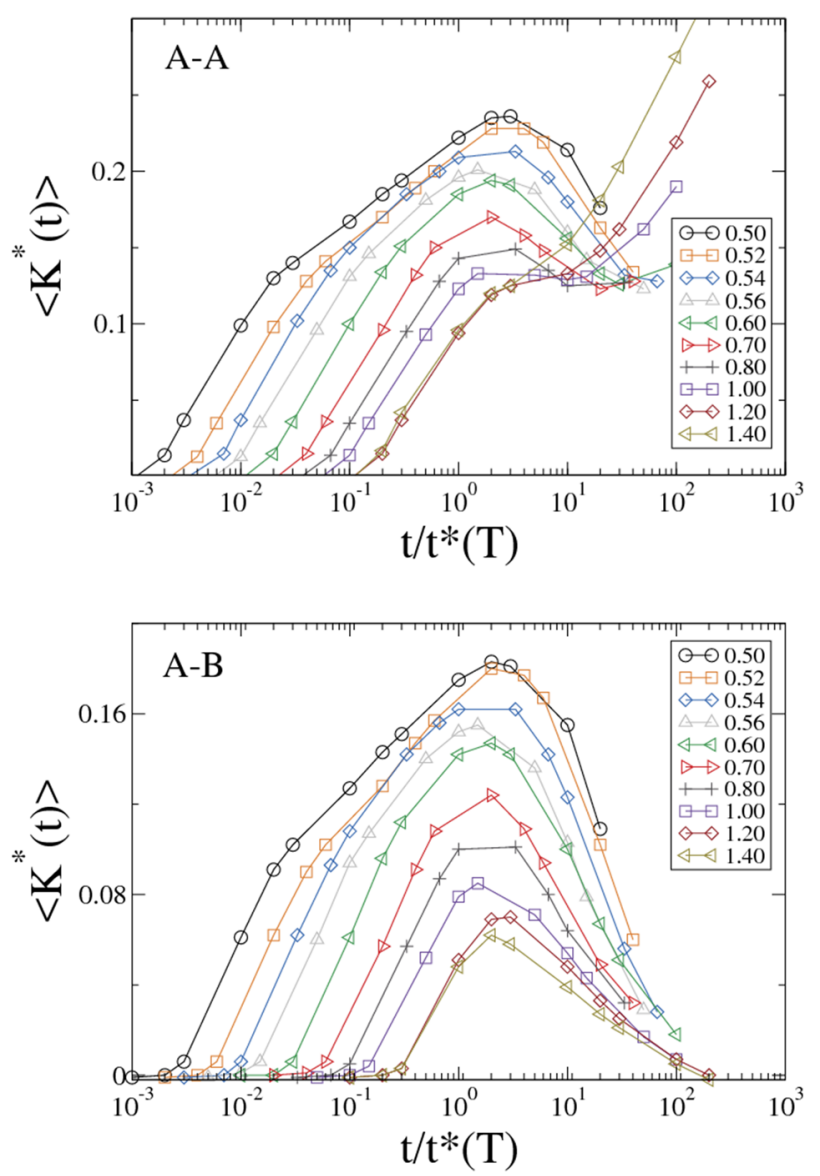

FIG. 9. Global correlation for $\mathrm{AA}$ and $\mathrm{AB}$ cases, at the nearest-neighbour distance, as a function of time relative to $t^{*}$ at each temperature. or they behave independently of each other. It was found that, within a single trajectory, the value of the displacement correlation function, $C_{\delta u}$, depends on the absolute value of the displacement (the displacement correlation between largedisplacement particles is higher than the average displacement correlation), so a similar behavior could be expected between propensity and Pearson's correlation. ${ }^{52}$ Figure 10 shows the average correlation at the nearest-neighbour distance calculated at $t^{*}\left(k_{i}\right)$ as well as the maximum correlation of a particle with its nearest-neighbours $\left(k_{i j_{\max }}\right)$, plotted as a function of the propensity $\left\langle\Delta \mathbf{r}_{i}^{2}\left(t^{*}\right)\right\rangle_{I C}$. There is no evident correlation between the two variables, meaning that a high correlation (HC) is not a necessary condition for a high propensity (HP). This can be ascribed to the fact that while propensity is a direct ICE-average of displacement, there is a subtle difference between the $C_{\delta u}$ function and $\mathrm{K}$ : The former is defined in terms of the difference between the displacement of a given particle and the mean displacement of all the particles, while Pearson's coefficient is defined in terms of the difference between the displacement of a particle in a single run with respect to the propensity of that same particle. Measuring correlations through Pearson's coefficient represents an alternative, complementary view of movement correlations, which do not depend on the absolute value of mobility,

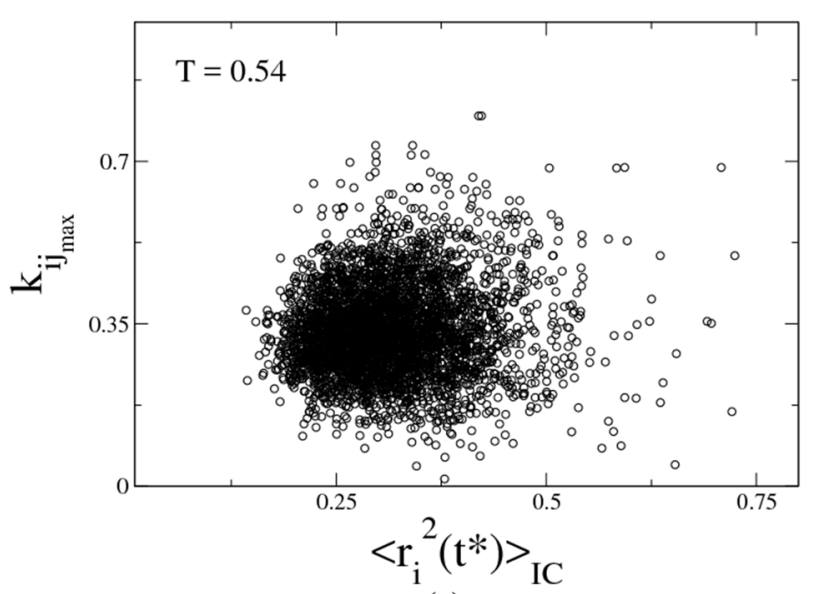

(a)

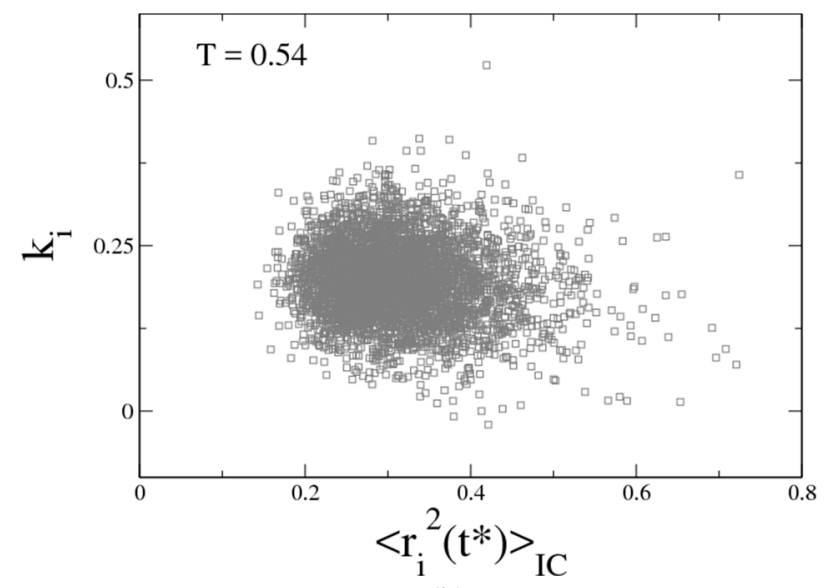

(b)

FIG. 10. Dispersion plots between the nearest-neighbour correlation and propensity. (a) Maximum correlation $\left(K_{i j \max }\left(t^{*}\right)\right)$ and (b) Average correlation $K\left(t^{*}\right)$. 
meaning that mobile particles need not to be correlated with their neighbours. In addition, Pearson's correlations can be related to the structure (initial configuration), as will be discussed in Sec. III E.

\section{E. Clusters of mobile and correlated monomers}

It has been established through MD simulations that particles with similar mobility (high or low) tend to form clusters. This was observed in single trajectories, where the particles that present the highest displacement form clusters and move cooperatively in a string-like fashion, ${ }^{11,12}$ giving rise to heterogeneous dynamics. When an ICE is considered, formation of similar-propensity clusters is also observed. ${ }^{25}$ In this section, we extend the definition of these clusters to include highly correlated particles. The following groups of monomers are defined: high correlation, or $\mathrm{HC}$, as the $7 \%$ of the monomers with the largest values of $k_{i}\left(t^{*}\right)$ of the $\mathrm{AB}$ case; high propensity, or $\mathrm{HP}$, as the $7 \%$ of monomers with the largest value of propensity; and low propensity, or LP, as the $7 \%$ with smallest propensity. Varying this percentage between 5 and 20 does not alter the conclusions of the analysis.

Each type of monomer was identified in the ICEs and the radial distribution function for different pairs was constructed. This analysis was performed from the 15 different ICEs generated (each one constructed from a different initial configuration) at each temperature, and the results are shown in Fig. 11. As a measurement of clustering, the coordination number of the first shell (up to the first minimum in the RDF) of each type of monomer pair is calculated, and the ratio with the general coordination number (from the RDF of all the monomers) is calculated,
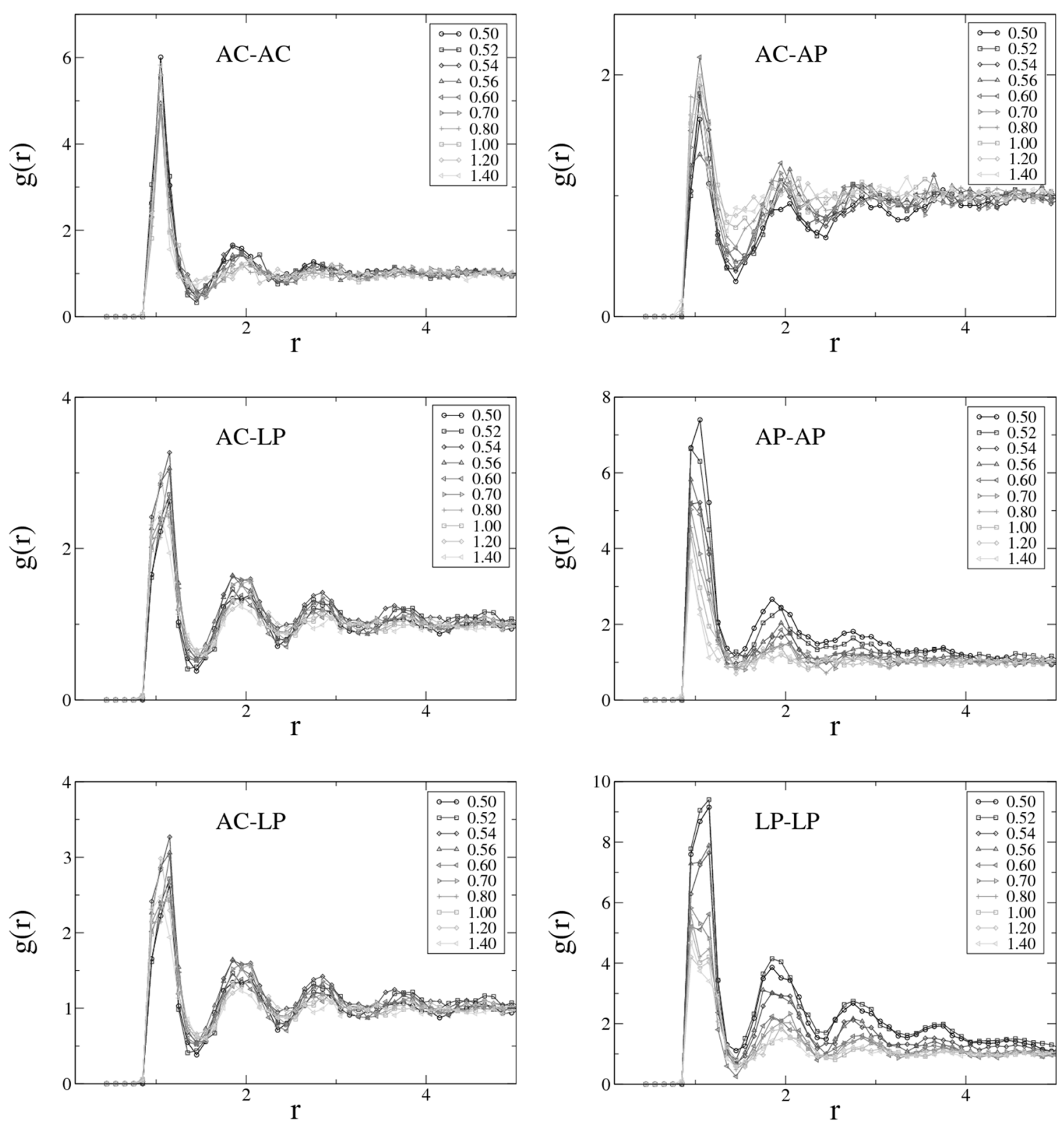

FIG. 11. Radial distribution functions between pairs of the different types of monomers: HC, HP, and LP. 


$$
f_{\text {cluster }}(T)=\frac{\rho(T) N_{i}(T)}{\rho_{i}(T) N(T)},
$$

where $\rho(T)$ and $\rho_{i}(T)$ are the densities of the whole system and the subsystem (HC, HP, or LP) at temperature T. $N(T)$ is the coordination number of the system, defined as the number of particles at the first shell up to the first minimum of the RDF, $N(T)=\int_{0}^{1.5} 4 \pi \rho g(r) r^{2} d r$, and $N_{i}$ is the average coordination number for a particular type. Figure 12 shows the variation of $f_{\text {cluster }}(T)$ with temperature for different monomer pairs. It is observed that alike monomers tend to cluster, especially LP monomers. This indicates that there are clusters of high-mobility particles and clusters of low-mobility particles, which is related to the concept of cooperative movement as explained before. The tendency to cluster for LP monomers increases significantly when temperature decreases and approaches $\mathrm{Tg}$.

Surprisingly, these types of monomers present a nonmonotonic behavior. The existence of a maximum has been observed for some dynamic correlation lengths, ${ }^{40}$ although there is some controversy regarding these results. But it has to be noted that our analysis of $f_{\text {cluster }}(T)$ describes only the clustering to first neighbors; this does not necessarily imply that the dynamic length of these zones presents the same behavior. It is interesting that the other two types of domains do not exhibit this behavior.

For the pair HP-LP, the cluster function is lower than one, indicating that they are structurally anti-correlated. The cluster function of the pair $\mathrm{HC}-\mathrm{HC}$ is significantly larger than one, indicating that monomers of this type also form clusters. HC-LP and HC-HP cluster functions are close to one at high temperatures; although close to Tg, the pair HC-LP show some tendency to form clusters, while the opposite is true for the pair HC-HP.

As a final remark, it can be mentioned that clustering of HC monomers, although it is significant, does not change appreciably with temperature. On the one hand, the magnitude of the dynamic correlation $\mathrm{K}(\mathrm{t})$ increases with the decrease of the temperature (as seen in Fig. 9), which means that movement of nearest-neighbours is, on average, more correlated, but on the other hand, clustering of correlated monomers remains the same. This increase in $\mathrm{K}(\mathrm{t})$ indicates that the dynamics

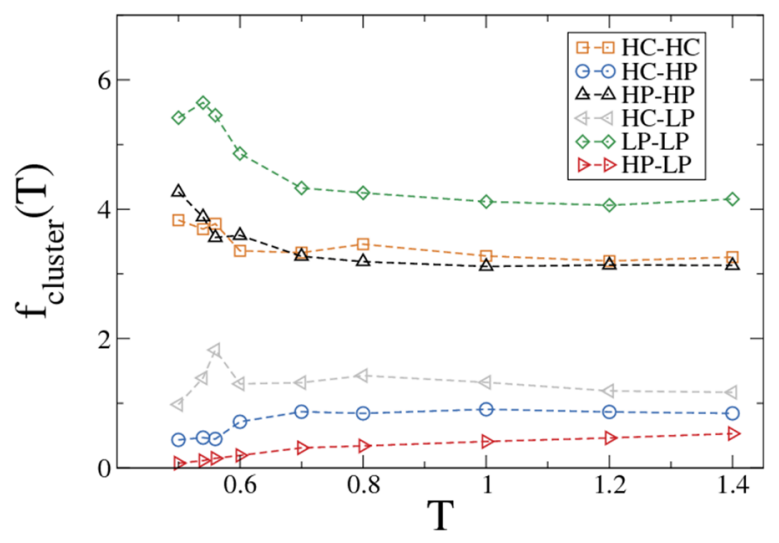

FIG. 12. The variation of $f_{\text {cluster }}$ with temperature for the different monomer pairs. It is evident that the same types of monomers tend to clusters. Error bars are the same size or smaller than the symbols.

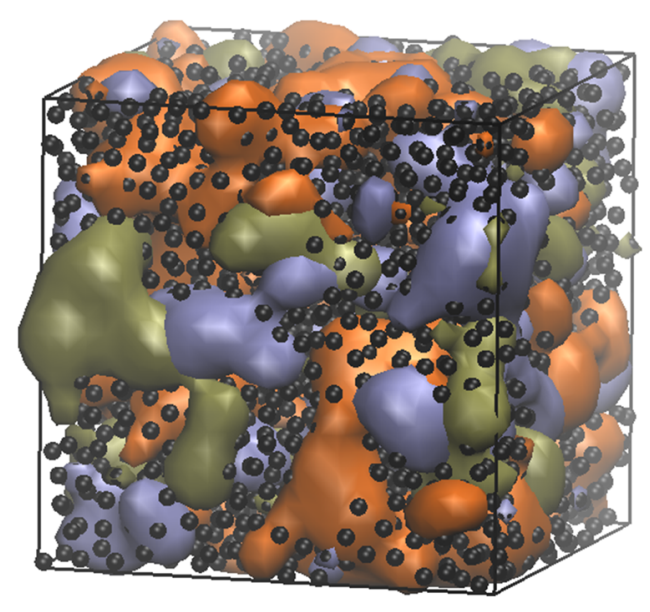

FIG. 13. Snapshot of an equilibrated system at a temperature of 0.54 . Monomers of different types are represented as isosurface maps, and small black spheres represent all the other monomers. The orange isosurface represents the HP, the green are the LP, and blue are the $\mathrm{HC}$.

is becoming more complex and more cooperative, which is one of the reasons behind the increase in relaxation times, but this does not imply that clustering of particles with high nearest-neighbour correlation increases.

In Fig. 13, a spatial configuration of the system at $\mathrm{T}=0.54$ is shown, where the positions of the different types of monomers are represented with isosurface maps in order to discriminate in a qualitative and simple manner the different zones in the system. Again, as suggested in Fig. 12, this new identification of particles, which have high correlation in an ICE, form clusters in a similar way to that occurring with highand low-mobility particles. An interesting observation is that each type of particles forms different clusters, in agreement with the fact that correlation and mobility are independent of each other as discussed before.

In Ref. 44, the authors discriminate two types of particle populations according to their propensity and their dynamic correlation with their neighbors. They implement a function, derived from an information theoretic approach, that measures the amount of correlation between two probability density functions of pairs of particles in the isoconfigurational ensemble. These probability distributions contain the absolute displacements of the particles in the whole set of trajectories of the IC. In our case, we take into account relative displacements with respect to the propensity of each particle using the Pearson coefficient. The relative displacement of the particles and then the correlation between pairs of particles allow us to define a new group or population of particles which emerge in clustered regions. This new group or population of particles, due to their correlated movement character, can be an alternative description of the cooperative relaxation regions.

\section{CONCLUSION}

The detailed dynamics of a linear polymer in the supercooled regime was studied by ICEM, which allows us to establish correlations between the initial configuration (structure) of the system and its dynamic behavior. Pearson's correlation 
coefficient, which is a measure of the correlation in particle displacement relative to its propensity, was used to analyze the dynamic correlation between monomers as a function of time and distance.

This work shows that this magnitude behaves similarly to the non-Gaussian parameter, and the time of maximum correlation corresponds to time $t^{*}$ of maximum dynamic heterogeneity. We also found that, in the temporal scale relevant to dynamic heterogeneity, the dynamic correlation between monomers is essentially the same for monomers belonging to the same chain or to different chains, except for a trivial higher correlation between bonded monomers. The general dynamic behavior is similar to that of simple liquids, showing that chain connectivity has no significant effect on dynamic behavior in supercooling conditions, at least in this non-entangled regime.

The main result of this work is that dynamic correlation in the ICE is independent of the monomer mobility: there is no statistical correlation between the values of Pearson's coefficient and propensity. As it happens with high-mobility and low-mobility particles, high-correlation particles tend to form clusters, but they do not form the same clusters. In this way, the dynamic correlation conditioned by the structure, measured by Pearson's coefficient applied to an ICE, represents an extra tool for characterizing heterogeneous dynamics in glass former that allows us to identify zones of highly correlated movement and complements the more traditional characterization in terms of mobility.

\section{ACKNOWLEDGMENTS}

Financial support from CONICET, ANPCyT, and UNMdP is gratefully acknowledged. This work used Mendieta cluster from CCAD-UNC, which is part of SNCAD-MinCyT, Argentina.

${ }^{1}$ P. G. Debenedetti and F. H. Stillinger, Nature 410, 259 (2001).

${ }^{2}$ L. Berthier and G. Biroli, Rev. Mod. Phys. 83, 587 (2011).

${ }^{3}$ A. Cavagna, Phys. Rep. 476, 51 (2009).

${ }^{4}$ S. Karmakar, C. Dasgupta, and S. Sastry, Rep. Prog. Phys. 79, 016601 (2016).

${ }^{5}$ G. Adam and J. H. Gibbs, J. Chem. Phys. 43, 139 (1965).

${ }^{6}$ T. R. Kirkpatrick, D. Thirumalai, and P. G. Wolynes, Phys. Rev. A 40, 1045 (1989).

${ }^{7}$ J.-P. Bouchaud and G. Biroli, J. Chem. Phys. 121, 7347 (2004).

${ }^{8}$ C. Donati, J. F. Douglas, W. Kob, S. J. Plimpton, P. H. Poole, and S. C. Glotzer, Phys. Rev. Lett. 80, 2338 (1998).

${ }^{9}$ M. Aichele, Y. Gebremichael, F. W. Starr, J. Baschnagel, and S. C. Glotzer, J. Chem. Phys. 119, 5290 (2003).

${ }^{10}$ Y. Gebremichael, M. Vogel, and S. C. Glotzer, J. Chem. Phys. 120, 4415 (2004).
${ }^{11}$ F. W. Starr, J. F. Douglas, and S. Sastry, J. Chem. Phys. 138, 12A541 (2013).

${ }^{12}$ Y. Gebremichael, T. B. Schrøder, F. W. Starr, and S. C. Glotzer, Phys. Rev. E 64, 051503 (2001).

${ }^{13}$ S. Sastry and C. Austen Angell, Nat. Mater. 2, 739 (2003).

${ }^{14}$ Y. Nie, X. Ye, Z. Zhou, W. Yang, and L. Tao, J. Chem. Phys. 141, 074901 (2014).

${ }^{15}$ S. Bernini and D. Leporini, J. Chem. Phys. 144, 144505 (2016).

${ }^{16}$ H. Tanaka, T. Kawasaki, H. Shintani, and K. Watanabe, Nat. Mater. 9, 324 (2010).

${ }^{17}$ H. Tanaka, Eur. Phys. J. E 35, 113 (2012).

${ }^{18}$ P. Charbonneau, E. Dyer, J. Lee, and S. Yaida, J. Stat. Mech.: Theory Exp. 2016, 074004.

${ }^{19}$ F. Sausset and G. Tarjus, Phys. Rev. Lett. 104, 065701 (2010).

${ }^{20}$ J. Taffs and C. Patrick Royall, Nat. Commun. 7, 13225 (2016).

${ }^{21}$ U. R. Pedersen, T. B. Schrøder, J. C. Dyre, and P. Harrowell, Phys. Rev. Lett. 104, 105701 (2010).

${ }^{22}$ B. Charbonneau, P. Charbonneau, and G. Tarjus, Phys. Rev. Lett. 108, 035701 (2012).

${ }^{23}$ R. L. Jack, A. J. Dunleavy, and C. P. Royall, Phys. Rev. Lett. 113, 095703 (2014).

${ }^{24}$ D. Chandler and J. P. Garrahan, Annu. Rev. Phys. Chem. 61, 191 (2010).

${ }^{25}$ A. Widmer-Cooper, P. Harrowell, and H. Fynewever, Phys. Rev. Lett. 93, 135701 (2004).

${ }^{26}$ A. Widmer-Cooper and P. Harrowell, J. Phys.: Condens. Matter 17, S4025 (2005).

${ }^{27}$ A. Widmer-Cooper and P. Harrowell, Phys. Rev. Lett. 96, 185701 (2006).

${ }^{28}$ A. Widmer-Cooper and P. Harrowell, J. Chem. Phys. 126, 154503 (2007).

${ }^{29}$ A. Widmer-Cooper and P. Harrowell, J. Non-Cryst. Solids 352, 5098 (2006).

${ }^{30}$ A. Widmer-Cooper, H. Perry, P. Harrowell, and D. R. Reichman, Nat. Phys. 4, 711-715 (2008).

${ }^{31}$ D. Coslovich and G. Pastore, J. Chem. Phys. 127, 124504 (2007).

${ }^{32}$ J. Ding, Y.-Q. Cheng, H. Sheng, and E. Ma, Phys. Rev. B 85, 060201 (2012).

${ }^{33}$ C. P. Royall and S. R. Williams, "The role of local structure in dynamical arrest," Phys. Rep. 560, 1 (2015).

${ }^{34}$ G. M. Hocky, D. Coslovich, A. Ikeda, and D. R. Reichman, Phys. Rev. Lett. 113, 157801 (2014).

${ }^{35}$ G. Biroli, J.-P. Bouchaud, A. Cavagna, T. S. Grigera, and P. Verrocchio, Nat. Phys. 4, 771 (2008).

${ }^{36}$ L. Berthier and W. Kob, Phys. Rev. E 85, 011102 (2012).

${ }^{37}$ G. M. Hocky, T. E. Markland, and D. R. Reichman, Phys. Rev. Lett. 108, 225506 (2012).

${ }^{38}$ G. Gradenigo, R. Trozzo, A. Cavagna, T. S. Grigera, and P. Verrocchio, J. Chem. Phys. 138, 12A509 (2013).

${ }^{39}$ G. Biroli and C. Cammarota, Phys. Rev. X 7, 011011 (2017).

${ }^{40}$ W. Kob, S. Roldán-Vargas, and L. Berthier, Nat. Phys. 8, 164 (2011).

${ }^{41}$ D. Ganapathi, K. H. Nagamanasa, A. K. Sood, and R. Ganapathy, Nat. Commun. 9, 397 (2018).

${ }^{42}$ E. Flenner and G. Szamel, Nat. Phys. 8, 696 (2012).

${ }^{43}$ B. Mei, Y. Lu, L. An, H. Li, and L. Wang, Phys. Rev. E 95, 050601 (2017).

${ }^{44}$ A. J. Dunleavy, K. Wiesner, R. Yamamoto, and C. P. Royall, Nat. Commun. 6, 6089 (2015).

${ }^{45}$ J. Colmenero, J. Phys.: Condens. Matter 27, 103101 (2015).

${ }^{46}$ G. S. Grest and K. Kremer, Phys. Rev. A 33, 3628 (1986).

${ }^{47}$ T. Yamazaki, J. Phys. Chem. B 118, 14687 (2014).

${ }^{48}$ S. Plimpton, J. Comput. Phys. 117, 1 (1995).

${ }^{49}$ W. Kob and H. C. Andersen, Phys. Rev. E 52, 4134 (1995).

${ }^{50}$ W. Götze, J. Phys.: Condens. Matter 11, A1 (1999).

${ }^{51}$ A. Rahman, Phys. Rev. 136, A405 (1964).

${ }^{52}$ F. Puosi and D. Leporini, J. Chem. Phys. 136, 164901 (2012). 